\title{
CORRIGENDUM
}

\section{The INECO Frontal Screening for the Evaluation of Executive Dysfunction in Cerebral Small Vessel Disease: Evidence from Quantitative MRI in a CADASIL Cohort from Colombia - Corrigendum}

Dorothee Schoemaker, Yesica Zuluaga, Anand Viswanathan, Markus D. Schirmer, Heirangi Torrico-Teave, Lina Velilla, Carolina Ospina, Gloria Garcia Ospina, Francisco Lopera, Joseph F. Arboleda-Velasquez and Yakeel T. Quiroz

doi: 10.1017/S1355617720000533. Published online by Cambridge University Press on 3 June 2020

In the original version of Schoemaker et al. (2020), Markus D. Schirmer's name was misspelled, and his affiliation was incorrect. The correct affiliation is the Department of Neurology, Massachusetts General Hospital, Harvard Medical School, Boston, MA 02114, USA.

The authors regret these errors. The original version has been updated.

\section{Reference}

Schoemaker, D., Zuluaga, Y., Viswanathan, A., Schirmer, M.D., Torrico-Teave, H., Velilla, L., Ospina, C., Garcia Ospina, G., Lopera, F., Arboleda-Velasquez, J.F., and Yakeel T. Quiroz. The INECO Frontal Screening for the Evaluation of Executive Dysfunction in Cerebral Small Vessel Disease: Evidence from Quantitative MRI in a CADASIL Cohort from Colombia. Journal of the International Neuropsychological Society. Published online 3 June 2020. doi: 10.1017/S1355617720000533. 